
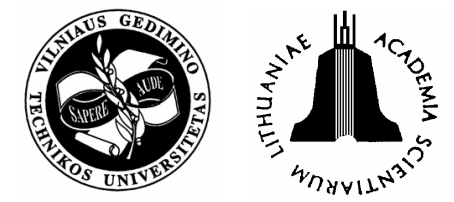

\title{
A MATHEMATICAL MODEL TO SET SPEED LIMITS FOR VEHICLES ON THE HIGHWAY
}

\author{
Sunday Ayoola Oke ${ }^{1}$, Ayokunle Bamigbaiye ${ }^{1}$, \\ Oluwafemi Isaac Oyedokun ${ }^{1}$, Oliver Ekpere Charles-Owaba ${ }^{2}$ \\ ${ }^{1}$ Dept of Mechanical Engineering, University of Lagos, Nigeria. \\ ${ }^{2}$ Dept of Industrial and Production Engineering, University of Ibadan, Nigeria. \\ E-mail: sa_oke@yahoo.com
}

Received 1 June 2006; accepted 4 September 2006

\begin{abstract}
The purpose of this paper is to develop a mathematical model that could guide certain speed limits for vehicles on roads. Excessive speeds on roads have been a major indirect cause of deaths on highways due to the sudden impact of vehicles that lead to accidents. The model presented here is developed on a measure of impact force of two vehicles engaged in a head-on collision. A hypothetical case study was investigated based on the impact of force on the human body. The results showed a variation of speed with occupation position distance. The paper also examines the role of seatbelt as a safety feature for vehicle occupants. Practically, developing a quantitative approach for evaluating the impacts of speed would help road regulatory authorities in reducing accidents and deaths on roads. It would also assist them in tracking "road traffic offenders" by setting controls to which road users must conform.
\end{abstract}

Keywords: speed limits, seatbelt, head-on collision, impact force, roads or highways.

\section{Introduction}

A speed limit can be defined as the maximum speed allowed by the regulatory authorities for vehicles using a particular highway. Speed limits are imposed on road highway users in order to prevent accidents and traffic fatalities.

Unfortunately, many other causes of accidents have been identified, such as:

1) the role of heavy drinking or alcohol on drivers' behaviour [1-3];

2) drug abuse among drivers [4];

3) other causes [5].

Researchers have argued that a common cause of road accidents is that no clear information is available on national levels on the prevalence of accidents due to driving under the influence of illicit drugs and drinking of alcohol. Thus, there is a high crash risk in drinking and driving and taking illicit drugs and driving [6].

In order to control speeds, regulatory authorities have installed various alternative approaches in order to attain results. One of these approaches is the use of road bumps, designed under conditions of variable speeds $[7,8]$. The study in paper [7] considers the conical-shaped road bump for theoretical and experimental analysis. The paper first determi- nes bump's height and width, as well as the effective distance between two consecutive road bumps. For the first relationship, tangent of angle of inclination of bump is proportional to the ratio of bump's height and width. For the second instance, the effective distance is proportional to the square of maximum speed and the acceleration component of the model. However, speed of vehicle at the side of speed and cosine of angle of inclination of bump side with road surface. The paper [8] is consistent with that described earlier.

Despite efforts by regulatory authorities to control speeds on roads, judgments on speed limits are usually intuitively made. This implies that variations in the implementation of road speed limits may be pronounced since no universal measurement exists. Therefore, the need to develop and test a mathematical model that would guide speed limits of vehicles on roads is important. With this, a scientific basis for setting speed limits would be in place. The speed limits proposed by this mathematical model help to keep the momentum change of vehicles within a range that will not constitute danger to vehicle occupation in case of a collision. Head-on collision was used in the model development for the worst-case scenario, as this will give the largest momentum change. Based on this impact force, the 
force required of the seatbelt to restrain the occupant in case of a collision is now evaluated. Other areas considered include the effect of the mass of the occupant, the distance travelled by collided vehicles on impact, distance of occupant from dashboard and whether or not the occupant used the seatbelt.

\section{Methodology}

Vehicles on highways move at high speed. While in an ideal case, the vehicles moving in opposite direction are separated by a dividing strip, they are not in many roads (in Nigeria for example). This makes it possible for fast moving vehicles to be involved in head-on collision. This will give the largest momentum change and consequently, force of impact on the occupant. If not using a seatbelt, the risk of injury is increased as the occupant's movement is unrestrained and will consequently be hurled forward by inertia.

The approach utilized is the law of conservation of linear momentum. This states that the total momentum of the bodies before impact is equal to the total momentum of bodies after impact. Using the change in kinetic energy or energy lost due to the impact the impact force can be calculated considering the distance travelled by the vehicles on impact (due consideration given to energy lost by sound during impact). The impact force is now used for other calculations, such as the restraining force of the seatbelt.

\subsection{Assumptions}

The problems of traffic can be very complex considering the infinite possibilities of events that can lead to a collision and what can follow. However, to obtain realistic result and simplify the problem, certain assumptions have to be made. These are:

- Only two of the vehicles on the highway are involved in the collision. If more than two vehicles collide, the impact analysis would be multibody, multi-directional, and therefore complex.

- The collision is head-on. This is to have a focus and an initial starting point.

- Energy is lost basically through work done by impacting force (crumbling both vehicles) and sound. Other sources of energy losses could be available in practice. However, they are treated as negligible to a research strategy and to avoid computational complexity.

- The vehicles, on impact, are "fixed" together and more through a distance with a common velocity before coming to rest.

- The impact force does not reach the occupant using a seatbelt when he/she is outside the crumbling zone (meant to dissipate energy due to impact force).

- The seatbelt acts as a rigid body when it holds the occupant in his seat against the pull of inertia on impact.

\subsection{Model development}

In developing a model that would practically represent the speed control model presented in this work, a number of notations are stated in this section, which would be utilized in the model development. The notations relate to the masses of the two vehicles with which collision occurs. Another important consideration is the velocities of the first and second vehicles as well as the common velocity after collision must have occurred. An important consideration is the issue of energy during collision. Here, it is known that there is energy change before and after collision. This energy change accounts for loss of energy through sound-wave propagation. Other aspects to consider are the area of impact during the collision process as well as the density of the vehicular body which is assumed to be mainly aluminium. An important consideration is also the common distance travelled on collision.

Thus, the following mathematical notations represent those discussed above in relation to the collision of the two vehicles:

$m_{1}$ - mass of $1^{\text {st }}$ vehicle;

$m_{2}-$ mass of $2^{\text {nd }}$ vehicle;

$v_{1}$ - initial velocity of $1^{\text {st }}$ vehicle;

$v_{2}$ - initial velocity of $2^{\text {nd }}$ vehicle;

$v_{3}$ - common velocity of the two vehicles from collision;

$\Delta E$ - energy lost through sound wave propagation;

$A$ - area of impact;

$\rho$ - density of vehicle body (aluminium);

$d t$ - common distance travelled on collision;

$d_{t}$ - a measure at the sense of the incidence;

$\tau$ - shear strength of the human bone;

$A$ - cross-sectional area of an average human bone assumed to be approximately circular annulus;

$d_{0}$ - distance of occupant from dashboard;

$F^{*}$ - inertia force on occupant due to impact;

$d$ - diameter of pin;

$w$ - width of belt;

$t$ - thickness of belt;

$m_{0}$ - mass of occupant;

$\sigma-$ strength of belt.

At the instance of collision, the concept of momentum is introduced. Since the vehicles are assumed to move in a linear and one-directional path, we can introduce the law of conservation of momentum to guide the collision. Thus, from the law of 
conservation of momentum, we then have equation 1 stated as follows:

$$
m_{1} v_{1}+m_{2}\left(-v_{2}\right)=\left(m_{1}+m_{2}\right) v_{3} \text {. }
$$

The negative velocity attached to the description of momentum for vehicle 2 indicates that the second vehicle moves in the opposite direction of the first. From equation (1), it is observed that the sum of the momentum before and after collision is the same. Before collision, the first vehicle has a specific mass and velocity. Also, the second vehicle has a different mass and velocity. However, the mass of the bodies after collision is the sum of the individual bodies. They also have a common velocity after collision.

Now, considering the energy balance, we would obtain an equation that shows the change in kinetic energy of the two bodies before and after collision on one side, and the energy changes in general as a result of the impact created on the other side of the equation. Therefore, the left-hand side of the equation would contain the kinetic energy of the moving first vehicle (A) plus the kinetic energy of the second vehicle (B) minus the kinetic energy of the collided vehicles moving together for a short period (see Fig 1). The right-hand side would contain the product of the force and distance as well as the energy lost through sound-wave propagation. Thus, equation shown below represents the mathematical expression for the energy balance:

$$
\begin{aligned}
& \frac{1}{2} m_{1} v_{1}^{2}+\frac{1}{2} m_{2} v_{2}^{2}-\frac{1}{2}\left(m_{1}+m_{2}\right) v_{3}^{2}= \\
& F_{1} d_{t}+\Delta E
\end{aligned}
$$

where $F_{1}$ - impact force.

Now, let us consider the two vehicles colliding. The length of the first vehicle (A) is given as $x_{1}$, while the distance between the extreme end of vehicle (A) and the end of vehicle (B) is $x_{2}$. The total length (i.e. $x_{1}+x_{2}$ ) is designated as $L$. This is shown in Fig 1.

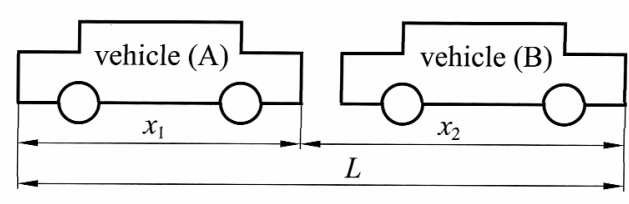

Fig 1. The "work done" analysis of colliding vehicles

We consider the energy lost by sound-wave propagation through each vehicle. Thus, we calculate the work done by each vehicle and the total work done by the vehicles. The work done by vehicle (A) is indicated as $W_{1}=F_{1} x_{1}$. For the second vehicle, the work done is $W_{2}=F_{1} x_{2}$. Therefore, the total work done, designated as $W_{T}$ is shown mathematically as $W_{T}=\Delta E=W_{1}+W_{2}$. The symbols $W_{1}$ and $W_{2}$ could be replaced by factors $F_{1}, x_{1}$ and $x_{2}$. Therefore, we have $W_{T}=\Delta E=W_{1}+W_{2}$. This leads us to $W_{T}=\Delta E=F_{1}\left(x_{1}+x_{2}\right)=F_{1} L$. However, we know that $F_{1}=m \frac{\Delta v}{t}=\rho A v \Delta v$.

Also, we know that:

$\Delta E=\rho A \nu \Delta v L$.

However, by substituting equation (3) for equation (2), we have:

$$
\begin{aligned}
& \frac{1}{2} m_{1} v_{1}^{2}+\frac{1}{2} m_{2} v_{2}^{2}-\frac{1}{2}\left(m_{1}+m_{2}\right) v_{3}^{2}= \\
& F_{1} d_{t}+\rho A v \Delta v L .
\end{aligned}
$$

Therefore:

$$
F_{1}=\frac{\frac{1}{2}\left(m_{1} v_{1}^{2}+m_{2} v_{2}^{2}\right)-\left(\frac{1}{2}\left(m_{1}+m_{2}\right) v_{3}^{2}+\rho A v \Delta v L\right)}{d_{t}} .
$$

It is assumed that this impact force will not reach an occupant using a seatbelt. In the design of the vehicle, the occupant will be positioned such that the energy associated with the impact force would have been dissipated in the vehicle crumple zone before it reaches him/her. An occupant who did not fasten his seatbelt will be hurled forward by inertia and will take this force on his body. Considering the force required to shatter a bone is based on its strength and the impact force from equation (4). Consider the transverse force acting on the bone as shown in Fig 2.

$$
2 \tau A \leq F_{1}^{\prime},
$$

where $F_{1}^{\prime}$ is the minimum force required to break the bone. The occupant will therefore sustain a fracture if:

$$
F_{1} \geq F_{1}^{\prime} \geq 2 \tau A \text {. }
$$

Therefore, to sustain a fracture:

$$
\frac{\frac{1}{2}\left(m_{1} v_{1}^{2}+m_{2} v_{2}^{2}\right)-\left(\frac{1}{2}\left(m_{1}+m_{2}\right) v_{3}^{2}+\rho A v \Delta v L\right)}{d_{t}} \geq 2 \tau A
$$

Fig 2. Transverse force acting on the bone

Variants of equation (7) are as follows:

- Vehicle 1 initially at rest: $v_{1}=0$. 


$$
\frac{\frac{1}{2} m_{2} v_{2}^{2}-\left(\frac{1}{2}\left(m_{1}+m_{2}\right) v_{3}^{2}+\rho A v_{3}^{2} L\right)}{d_{t}}=F_{1} .
$$

- Vehicle 2 initially at rest: $v_{2}=0$.

$$
\frac{\frac{1}{2} m_{1} v_{1}^{2}-\left(\frac{1}{2}\left(m_{1}+m_{2}\right) v_{3}^{2}+\rho A v_{3}^{2} L\right)}{d_{t}}=F_{1} .
$$

- Both vehicles are brought to rest after impact: $v_{3}=0$.

$$
\frac{\frac{1}{2}\left(m_{1} v_{1}^{2}+m_{2} v_{2}^{2}\right)}{d_{t}}=F_{1} .
$$

- If all the energy lost is due to work done by impact force i.e. no energy lost by sound wave propagation.

$$
\begin{gathered}
\rho A v_{3}^{2} L=0 ; \\
F_{1}=\frac{\frac{1}{2}\left(m_{1} v_{1}^{2}+m_{2} v_{2}^{2}\right)-\frac{1}{2}\left(m_{1}+m_{2}\right) v_{3}^{2}}{d_{t}} .
\end{gathered}
$$

Alternatively, the analysis can be done based on the occupant himself and not on the vehicles. Consider an occupant of mass $m_{0}$ inside vehicle 1 moving with an initial velocity $v_{1}$. Initially, it is assumed for the purpose of analysis that the occupant is not using the seatbelt. The velocity of the occupant on impact $v_{2}=0$. This is because he will be hurled against the dashboard and suddenly brought to rest.

Therefore:

$$
\begin{aligned}
& \frac{1}{2} m_{0} v_{1}^{2}-\frac{1}{2} m_{0} v_{2}^{2}=F d_{0} ; \\
& v_{2}=0 ; \\
& \frac{1}{2} m_{0} v_{1}^{2}=F d_{0},
\end{aligned}
$$

where $F d_{0}$ - work done in bringing occupant to rest.

$$
F=\frac{m_{0} v_{1}^{2}}{2 d} .
$$

Alternatively, if the occupant was in vehicle 2 moving with an initial velocity $v_{2}$, the force of impact on the occupant will be:

$$
F=\frac{m_{0} v_{2}^{2}}{2 d} .
$$

It is obvious from equations (13) and (14) that the force on the occupant depends directly on the product of his mass and velocity before impact and inversely proportional to his distance from the dashboard. Based on this force and the force to cause fracture, the velocity of the vehicle to cause injury on collision can be estimated. Recall that the mini- mum force required to cause fracture is given by $F_{1}^{\prime} \geq 2 \tau A$.

If $F \geq F_{1}^{\prime}$, bone fractures.

Therefore: $\frac{m_{0} v_{1}^{2}}{2 d_{0}} \geq 2 \tau A$.

Therefore:

$$
\begin{aligned}
& v_{1} \geq \sqrt{\frac{4 \tau A d_{0}}{m_{0}}} ; \quad v_{1} \geq v_{1}^{*} ; \\
& v_{1}^{*}=\sqrt{\frac{4 \tau A d_{0}}{m_{0}}} .
\end{aligned}
$$

This gives the limiting velocity of the vehicle that will prove hazardous on impact for a commuter travelling without using the seatbelt. A speed limit can therefore be obtained using equation (15). Now assuming the occupant uses a seatbelt as shown below, the restraining force and consequently strength of the belt that will not fail in event of an impact can be estimated. If we consider the sitting position of the occupants before the impact, then the forces on the seatbelt (this is an inextensible fabric which in configuration will act as a rigid body), i. e. the free body diagram of the forces acting is as shown in Fig 3.

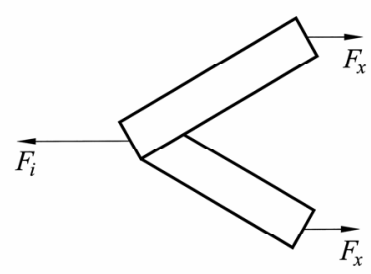

Fig 3. Free body diagram of the forces acting

Therefore:

$$
\begin{aligned}
& F_{i}=2 F_{x} ; \\
& F^{*}=2 F_{x} ;
\end{aligned}
$$

$$
F_{i}=\frac{m_{0} v_{i}^{2}}{2 d} ; \quad \frac{m_{0} v_{i}^{2}}{2 d}=2 F_{x} .
$$

Therefore:

$$
F_{x}=\frac{m_{0} v_{i}^{2}}{4 d} .
$$

Equation (17) gives the minimum force the seatbelt must be able to withstand at the pinned ends to be able to restrain the occupant in the event of an impact. Taking a section of the belt through one of its pinned ends, we will have the strength of the seatbelt defined by $\sigma A=F_{x}$.

Therefore (see Fig 4):

$$
\sigma=\frac{F_{x}}{A}=\frac{F_{x}}{t(w-d)} \text {. }
$$




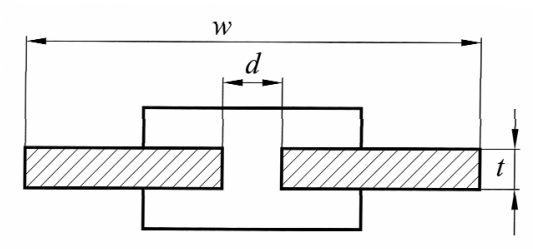

Fig 4. Seatbelt scheme

Equating (17) and (18):

$$
\sigma=\frac{m_{0} v_{i}^{2}}{4 d_{0} t(w-d)} \text {. }
$$

Recall that the velocity to cause fracture is given by equation as:

$$
v_{1}^{*}=\sqrt{\frac{4 \tau A d_{0}}{m_{0}}} .
$$

Setting $v_{1}=v_{1}^{*}$ to obtain a minimum belt strength for the occupant's safety in the event of a collision, we will have:

$$
v_{1}=v_{1}^{*}=\sqrt{\frac{4 \tau A d_{0}}{m_{0}}} .
$$

Therefore (from equation (19)):

$$
\sigma=\frac{m_{0} v_{i}^{* 2}}{4 d_{0} t(w-d)} \text {. }
$$

Putting (21) into (22), we will have:

$$
\sigma=\frac{m_{0} \frac{4 \tau A d_{0}}{m_{0}}}{4 d_{0} t(w-d)}=\frac{\tau A}{t(w-d)} .
$$

Therefore:

$$
\sigma=k \tau \text {, }
$$

where

$$
k=\frac{A}{t(w-d)} .
$$

From equation (24), the strength of the belt can be designed based on the strength of the bone of the occupant. An average value for the shear strength of a healthy human being can be used, due consideration given to $k$ (avec ratio). Equation (22) gives a relationship between the strength of the belt and the velocity of the vehicle. These can be related together keeping all other parameters constant. Same can be done for the strength of the belt and the mass of the occupant.

Based on the shear strength of the human bone and the value of $k$, equation (24), the minimum value of $\sigma$ for safety can be set. This is now utilized in equation (22) to obtain the speed limit on the highway.

\section{Case study}

Consider a hypothetical value for the shear strength of the human bone say $\tau=400 \mathrm{kN} / \mathrm{m}^{2}$. If $k$ is taken as 4 i.e. the bone of a typical bone in a man say Femur is 4 times the cross-section of the seatbelt. The outer diameter of the Femur is taken as $4 \mathrm{~cm}$ with a thickness of $1 \mathrm{~cm}$. The Femur is thus as shown in Fig 5.

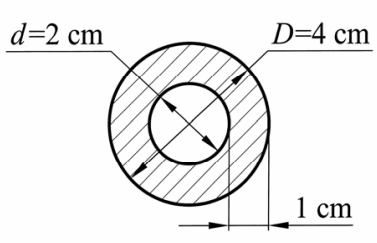

Fig 5. Femur scheme

$$
A=\pi \frac{\left(D^{2}-d^{2}\right)}{4} \text {. }
$$

Therefore:

$A=\pi \frac{\left(4^{2}-2^{2}\right)}{4}=9.428 \mathrm{~cm}^{2}=9.428 \cdot 10^{-4} \mathrm{~m}^{2}$

and

$$
k=4=\frac{A}{t(w-d)} .
$$

Therefore:

$$
\tau(w-d)=\frac{A}{4}=\frac{9.428 \cdot 10^{-4}}{4}=2.36 \cdot 10^{-4} \mathrm{~m}^{2} \text {. }
$$

Put (29) into (22)

$$
\sigma=\frac{m_{0} v_{i}^{* 2}}{4 d_{0} \cdot 2.36 \cdot 10^{-4}}=\frac{\left(1.06 \cdot 10^{3}\right) m_{0} v_{i}^{* 2}}{d_{0}}
$$

Equation (30) expresses the strength of the belt $\sigma$ as a function of mass of occupant $m_{0}$, distance of occupant $d_{0}$ from the dashboard, $v_{1}^{*}$ velocity of the vehicle (and of course the occupant).

Rewrite equation (24) with values:

$$
\sigma=k \tau=4 \cdot 400 \cdot 10^{3}=1.6 \cdot 10^{6} \mathrm{~N} / \mathrm{m}^{2} \text {. }
$$

Equating (30) and (31) we will have:

$$
\begin{aligned}
& \frac{\left(1.06 \cdot 10^{3}\right) m_{0} v_{i}^{* 2}}{d_{0}}=1.6 \cdot 10^{6} \text {, or } \\
& v_{1}^{*}=38.85 \sqrt{\frac{d_{0}}{m_{0}}} .
\end{aligned}
$$

Equation (32) expresses the speed limit as a function of the occupant mass and his distance from the dashboard. The variation of $v_{1}^{*}$ with these parameters can therefore be observed. 
Case 1: Variation of speed with mass (distance is fixed)

Assuming $d_{0}=0.5 \mathrm{~m}$.

Therefore:

$$
v_{1}^{*}=38.85 \sqrt{\frac{0.5}{m_{0}}}=\frac{27.47}{\sqrt{m_{0}}} .
$$

Considering occupants with various mass range, as shown below:

$$
30 \leq m_{0} \leq 70 \text {. }
$$

The result can be expressed in a compact form in the Table 1 .

Table 1. Variation of speed with masses (distance is fixed)

\begin{tabular}{|c|c|c|c|c|}
\hline$i$ & $m_{0}, \mathrm{~kg}$ & $\sqrt{m_{0}}$ & $\left(\sqrt{m_{0}}\right)^{-1}$ & $v_{1}^{*}, \mathrm{~m} / \mathrm{s}$ \\
\hline 1 & 30 & 5.48 & 0.183 & 5.02 \\
2 & 40 & 6.32 & 0.158 & 4.34 \\
3 & 50 & 7.07 & 0.141 & 3.88 \\
4 & 60 & 7.74 & 0.130 & 3.55 \\
5 & 70 & 8.37 & 0.120 & 3.28 \\
\hline
\end{tabular}

Case 2: Variation of speed with occupant position distance $d_{0}$

Mass is fixed, therefore $\left(m_{0}=60 \mathrm{~kg}\right)$.

Therefore:

$$
v_{1}^{*}=38.85 \sqrt{\frac{d_{0}}{60}}=5.02 \sqrt{d_{0}} .
$$

The results can be expressed in a compact form in the Table 2.

Table 2. Variation of speed with occupant position distance $d_{0}$

\begin{tabular}{|c|c|c|c|}
\hline$i$ & $d_{0}, \mathrm{~m}$ & $\sqrt{d_{0}}$ & $v_{1}^{*}, \mathrm{~m} / \mathrm{s}$ \\
\hline 1 & 0.5 & 0.707 & 3.55 \\
2 & 0.6 & 0.775 & 3.89 \\
3 & 0.8 & 0.837 & 4.20 \\
4 & 0.8 & 0.894 & 4.50 \\
5 & 1.0 & 1.000 & 5.20 \\
\hline
\end{tabular}

\section{Discussion of results}

The case study validates the fact that the speed limit of a vehicle can be set based on the mass of the occupant or on the distance of the occupant from the dashboard.

The variation of the speed limit with the aforementioned parameters are as shown on the plots above.

The first plot shows that for increasing occupant mass, the velocity has to be reduced (i.e. lower speed limits). On the other hand, the speed limit can be higher for higher values of do (i.e. occupant distance from dashboard).

\section{Conclusions}

This paper has been able to develop a mathematical model that determines the speed limit of vehicles on the highway based on parameters relating to the occupant and his position in the vehicle.

The model is based on the fact that the strength of a seatbelt required to hold an occupant (restrain him on his seat) in the event of a collision is proportional (directly) to its inertia force.

This was used in the model to determine the strength of the belt as a function of other parameters relating to the geometry of the belt, but material and position of the occupant in the vehicle.

The model shows that the speed limit can be set using occupant mass or position in vehicle as criterion. The case study cited justifies this.

The scope of the paper may be widened by considering multiple vehicle collision with each vehicle containing $n$ occupants $(n>1)$.

\section{References}

1. Kennedy, B. P.; Isaac, N. E.; Graham, J. D. The role of heavy drinking in the risk of traffic fatalities. Risk Analysis, Vol 16, No 4, 1996, p. 565-569.

2. Mayou, R.; Bryant, B. Alcohol and road traffic accidents. Alcohol, Vol 30, No 6, 1995, p. 709-711.

3. Chipman, M. L., Macdonald, S., Mann, R. E. Being "at fault" in traffic crashes: does alcohol, cannabis, cocaine, or polydrug abuse make a difference? Injury Prevention, Vol 9, No 4, 2003, p. 343-348.

4. Macdonald, S.; Mann, R. E., Chipman, M. AnglinBodrug, K. Collision and traffic violations of alcohol, cannabis and cocaine abuse clients before and after treatment. Accident Analysis and Prevention, Vol 36, No 5, 2004, p. 795-800.

5. Chipman, M. L. Risk factors for injury: similarities and differences for traffic crashes and other sauses. Accident Analysis and Prevention, Vol 27, No 5, 1995, p. 699-706.

6. Vanlaar, W.; Yannis, G. Perception of road accident sauses. Accident Analysis and Prevention, Vol 38, 2006, p. 155-161.

7. Oke, S. A.; Salau, T. A. O.; Adeyefa, A. O. Vehicle speed control using road bumps: Part 2. Transport, Vol XX, No 3, 2005, p. 99-105.

8. Salau, T. A. O.; Adeyefa, A. O.; Oke, S. A. Vehicle speed control using road bumps. Transport, Vol XIX, No 3, 2004, p. 130-136. 